\title{
A rare cause of acute pulmonary oedema
}

\author{
Samir A Khwaja, Umasuthan Srirangalingam and William M Drake
}

\section{Case presentation}

A 38-year-old Afro-Caribbean woman presented to her local hospital with a two-year intermittent history of shortness of breath, cough productive of pink frothy sputum and palpitations. She had a background of congenital mitral stenosis with xenograft replacement at age 11, followed by prosthetic mitral valve replacement 4 years later. Over a period of six months, she had five hospital admissions with similar symptoms, which were attributed to recurrent severe pulmonary oedema and, on one occasion, associated with an episode of sustained ventricular tachycardia (VT). On each occasion, her symptoms responded to treatment with diuretics and nitrates. She was on lifelong warfarin therapy. She had a strong family history of diabetes and hypertension and smoked 10 cigarettes a day.

On examination, the patient was tachypnoeic and sweaty with a pulse rate of 140 beats per minute and a blood pressure of 190/120 mmHg. Cardiac examination revealed a collapsing pulse and a loud prosthetic first heart sound with a soft diastolic murmur. She also had coarse crepitations at both lung bases.

Laboratory testing demonstrated a haemoglobin of $9.7 \mathrm{~g} / \mathrm{dl}$ and a borderline low mean corpuscular volume (MCV), but a subsequent serum ferritin test was normal. Haemoglobin electrophoresis was normal. She had a leucocytosis of $26.4 \times 10^{9} / 1$ and her C-reactive protein was $183 \mathrm{mg} / \mathrm{l}$. Her renal function was mildly impaired. Chest radiograph showed an enlarged heart, sternotomy wires and a prosthetic mitral valve. Electrocardiogram (ECG) showed sinus rhythm with left ventricular hypertrophy and strain pattern. An echocardiogram during the patient's last admission had shown good left ventricular function, normal function of the mitral valve prosthesis and moderate aortic regurgitation.

\section{Differential diagnosis}

The patient presented with shortness of breath and a productive cough, tachycardia, hypertension and important auscultatory findings. She had five previous admissions and on each occasion had been diagnosed with pulmonary oedema. Other diagnoses that were considered included infective endocarditis, pneumonia and an infective exacerbation of chronic obstructive pulmonary disease. There were no clinical signs to suggest endocarditis and the radiographic findings were consistent with pulmonary oedema,

Samir A Khwaja, foundation year 1 (FY1) trainee in endocrinology; Umasuthan Srirangalingam, NIHR clinical lecturer in endocrinology; William M Drake, professor of endocrinology and consultant physician

Department of Endocrinology, St Bartholomew's Hospital, London, UK thought to be cardiogenic in origin. As the patient had a prosthetic valve, valve leakage resulting in pulmonary oedema was a key concern. Other explanations for her acute pulmonary oedema included non-cardiogenic causes such as acute lung injury secondary to pneumonia or sepsis, fluid overload resulting from renal impairment, the result of hypertensive crisis or, unusually, a neurogenic cause including an undetected seizure or cerebral insult. The aortic regurgitation seen on her echocardiogram was attributed to her longstanding hypertension. No vegetations were seen echocardiographically to suggest infective endocarditis.

\section{Initial management}

The patient was treated for acute pulmonary oedema with oxygen, a loop diuretic and nitrate therapy. Her hypertension and tachycardia persisted despite this treatment.

\section{Case progression}

Given the recent trans-thoracic echocardiographic findings, a trans-oesophageal echocardiogram (TOE) was performed to better visualise the prosthetic valve. This demonstrated a paraprosthetic jet of mitral regurgitation of moderate severity, aortic regurgitation of moderate severity and a globally hypertrophied left ventricle with moderate to good function. A coronary angiogram revealed unobstructed vessels. The precipitant remained unclear. As the findings were felt to be insufficient to explain the clinical presentation, alternative causes of the pulmonary oedema were considered. Thyroid function tests were normal. Measurements of urinary catecholamines were requested during the convalescent phase of her illness. These revealed an elevated noradrenaline of $3911 \mathrm{nmol} / 1 / 24$ hours (normal range (NR) $<560 \mathrm{nmol} / \mathrm{l}$ ) with normal dopamine and adrenaline levels, suggesting the likely diagnosis of a phaeochromocytoma. On further questioning, the patient reported a long history of sweating and palpitations.

Abdominal and pelvic computed tomography (CT) revealed an enhancing mass, measuring $6.5 \times 4.5 \mathrm{~cm}$, in the lower left para-aortic region just above the level of the aortic bifurcation, consistent with a paraganglioma (Fig 1B). There was no evidence

\section{Key learning points}

- Paragangliomas often present with non-specific features that mimic more common conditions, which could lead to a delayed diagnosis.

- Diagnosis can be reliably confirmed by demonstrating elevated blood and urine levels of metanephrines and/or catecholamines.

- Patients with paragangliomas require careful pre-operative management, genetic testing and lifelong follow-up. 
of adrenal enlargement or metastatic disease. Functional imaging using radiolabelled metaiodobenzylguanidine ( ${ }^{123}$ I-MIBG) showed a focal area of intense uptake in the same position, confirming the diagnosis (Fig 1C-D).

Intravenous blockade with phenoxybenzamine was followed by beta blockade with propranolol. Pre-operative preparation included cessation of warfarin therapy and antibiotic prophylaxis to reduce the risk of infective endocarditis. The tumour was resected without complication and the patient made a good recovery. Histological examination revealed a large wellcircumscribed highly vascular tumour consistent with a paraganglioma. There were focal regions of infiltration at the border and in the lymphovascular space, suggesting a potentially aggressive phenotype.

Given the histology, a single treatment dose of ${ }^{131}$ I-MIBG was administered post-operatively. Repeat urinary catecholamines at six months showed levels within the normal range. The patient has since been reviewed annually in the endocrine clinic and she remains well, with urinary metanephrines consistently within the normal range. Genetic testing was undertaken to rule out a familial syndrome. This revealed a heterozygous sequence variant (c.-38G $>A$ ) in the $5^{\prime}$ untranslated region (UTR) of the succinate dehydrogenase $\mathrm{C}$ (SDHC) gene, although the pathological significance of this variant awaits confirmation with functional studies of the tumoural RNA.

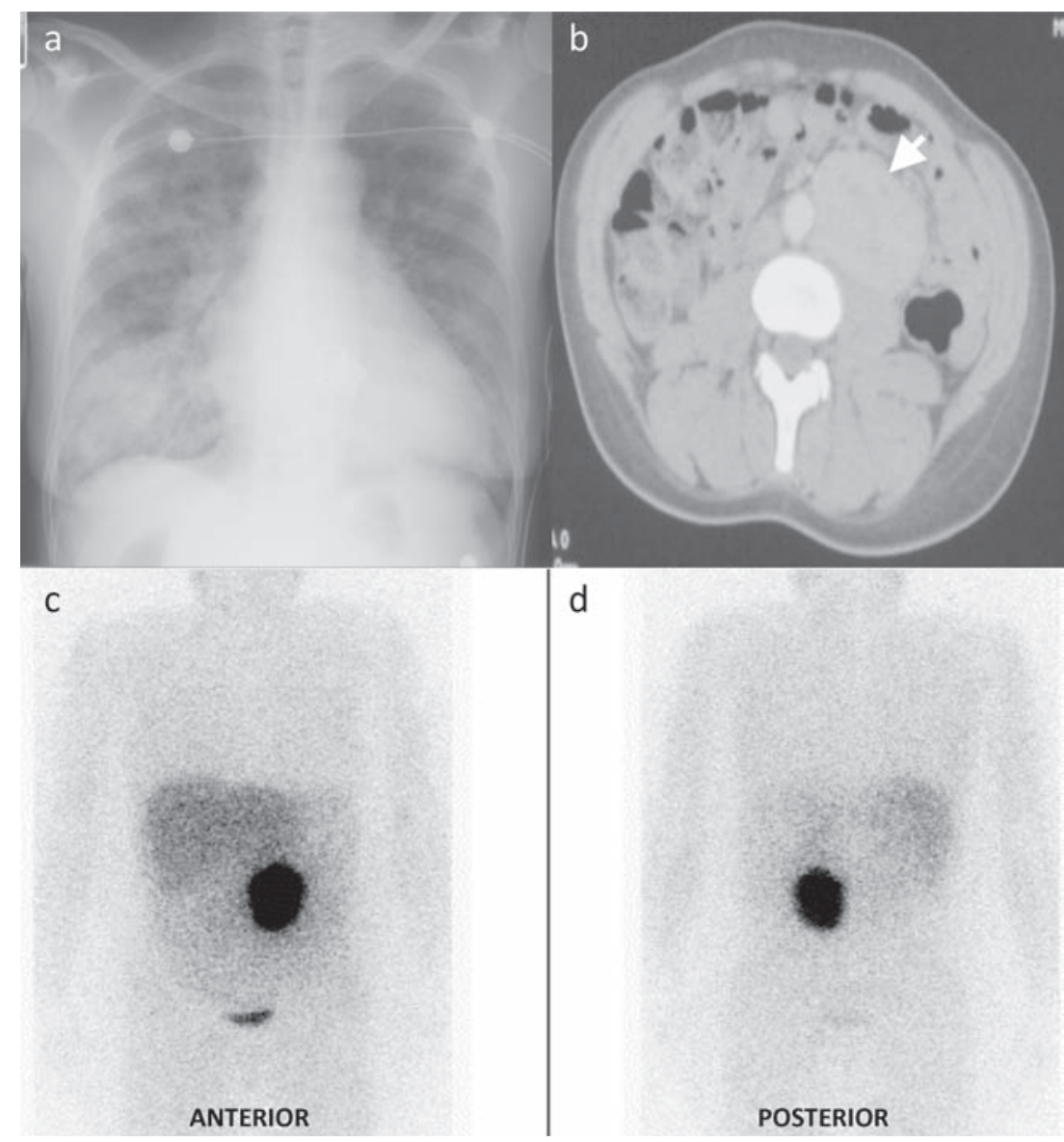

Fig 1. Imaging to investigate the causes of pulmonary oedema. (a) Chest radiograph on admission. (b) CT abdomen, demonstrating a left para-aortic mass (white arrow). (c) and (d) ${ }^{123}$ I-MIBG scan with anterior and posterior view, respectively, at 24 hours post-isotope infusion, demonstrating uptake.

\section{Discussion}

Paragangliomas are rare tumours composed of chromaffin tissue, which originate from sympathetic and parasympathetic ganglia at extra-adrenal sites. They comprise approximately $10-20 \%$ of all chromaffin tumours. Up to a third of paragangliomas occur as part of a familial syndrome. ${ }^{1}$ The majority of catecholamine-secreting paragangliomas are found in the abdomen and cause symptoms that can vary greatly. Patients typically present with headache, palpitations, sweating and often paroxysmal or sustained hypertension. ${ }^{2}$ If unrecognised, lifethreatening sequelae of catecholamine excess, including myocardial infarction, congestive heart failure and even cardiovascular collapse can develop.

Our patient presented on multiple occasions with frank pulmonary oedema and symptoms of cardiac failure that were out of keeping with the assessment of the severity of her cardiac lesion, raising suspicion of a separate, non-cardiogenic contributor to her illness. In this case, catecholamine release from the paraganglioma caused marked peripheral vasoconstriction, leading to decompensation of an already abnormal heart.
Surgical excision is the treatment of choice for paragangliomas. Pre-operative preparation is essential, however, to avoid the lifethreatening complications of catecholamine excess. Irreversible $\alpha$-blockers, such as phenoxybenzamine, given prior to surgery relieve $\alpha$-mediated vasoconstriction. A $\beta$-blocker can be added if the patient is tachycardic but should never be started before $\alpha$-blockade as the loss of $\beta$-adrenoceptor-mediated vasodilatation would leave $\alpha$-adrenoceptor stimulation unopposed, leading to a hypertensive crisis. It has been shown that effective blockade and then tumour resection can reverse many of the echocardiographic features of cardiac failure seen in these patients.

Our patient had a large paraganglioma that was successfully resected. Despite concern over the initial histological characteristics, there has been no evidence of metastatic disease on followup. Predictors for malignant disease include an initial tumour that is larger than $5 \mathrm{~cm}$ in diameter, an extra-adrenal location and tumours that are associated with mutations in succinate dehydrogenase B (SDHB). ${ }^{3,4}$ In our patient, a potential mutation in the SDHC gene has been noted. These mutations are associated with a low risk of malignancy. To date, defects in 11 genes have been associated with these tumours in approximately a third of patients studied, making genetic testing routine practice 
in patients under the age of 50 years. ${ }^{5}$ Knowledge of an associated mutation can guide future follow-up regimes and allow familial screening.

This case illustrates the protean manifestations of chromaffin tumours. The true underlying diagnosis was obscured by the very plausible initial explanation of cardiac failure secondary to prosthetic valvular dysfunction. It also highlights the need for the physician to consider the rare causes of common presentations, particularly when investigations are inconsistent with the presumed diagnosis. In cases of catecholamine excess, the preceding history is often instructive. Alpha blockade remains the key intervention in stabilising such patients.

\section{References}

1 Amar L, Bertherat J, Baudin E et al. Genetic testing in pheochromocytoma or functional paraganglioma. J Clin Oncol 2005;23:8812-8.
2 Lenders JW, Eisenhofer G, Mannelli M, Pacak K. Phaeochromocytoma. Lancet 2005;366:665-75.

3 Ayala-Ramirez M, Feng L, Johnson MM et al. Clinical risk factors for malignancy and overall survival in patients with pheochromocytomas and sympathetic paragangliomas: primary tumor size and primary tumor location as prognostic indicators. J Clin Endocrinol Metab 2011; 96:717-25.

4 Amar L, Baudin E, Burnichon N et al., Succinate dehydrogenase B gene mutations predict survival in patients with malignant pheochromocytomas or paragangliomas. J Clin Endocrinol Metab 2007;92:3822-8.

5 Jafri M, Maher ER. The genetics of phaeochromocytoma: using clinical features to guide genetic testing. Eur J Endocrinol 2011;166:151-8.

Address for correspondence: Dr SA Khwaja,

St Bartholomew's Hospital (Barts), West Smithfield, London EC1A 7BE.

Email: samirkhwaja@doctors.org.uk

\section{Gifts and memorabilia}

A new range of gifts based on a huge collection of art, and artifacts housed at the RCP's home in Regent's Park.

Contemporary mugs

Colourful, heavyweight earthenware mugs.

Available in red (pictured), blue and grey.

$£ 9.00$ UK, $£ 12.00$ overseas (inc $p+p$ )

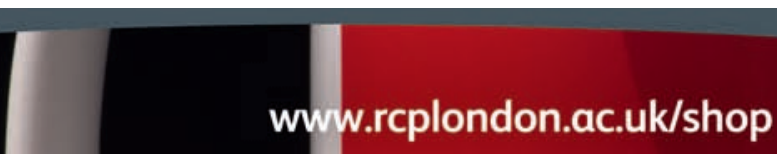

(1)

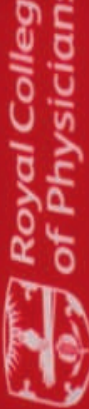

